\section{DATACIONES AMS \\ DE LA CUEVA DE TITO \\ BUSTILLO (ASTURIAS) (1)}

\author{
ACCELERATOR MASS SPECTROMETRY \\ DATES OF TITO BUSTILLO CAVE \\ (ASTURIAS, SPAIN)
}

ALFONSO MOURE ROMANILLO (*)

\section{RESUMEN}

Dataciones $\mathrm{C} 14$ por acelerador de las áreas de actividad de la cueva de Tito Bustillo permiten apoyar algunas reflexiones sobre el modelo teórico de periodización del Magdaleniense.

\begin{abstract}
Accelerator Mass Spectrometry dates from the activity areas of Tito Bustillo cave support some thoughts on the theoretical model of Magdalenian chronology.
\end{abstract}

Palabras-clave: C14 convencional. AMS-C14. Magdaleniense. Arte rupestre paleolítico.

Key words: Radiocarbon. Accelerator. Magdalenian. Paleolithic cave art.

\section{INTRODUCCIÓN}

Las primeras excavaciones en la cueva de Tito Bustillo (Ribadesella, Asturias) (Fig.1) se remon-

(*) Universidad de Cantabria. 39005 Santander. Correo electrónico: mourea@ccaix3.unican.es

El artículo fue remitido en su versión final el 22-X-97.

(1) Las dataciones que son el apoyo principal de este trabajo forman parte del proyecto de investigación PS92-0137 de la DGICYT (Documentación del arte rupestre en el sector central de la costa cantábrica: una evaluación de técnicas de trabajo). Para los gastos derivados de la datación se contó con una subvención adicional del Vicerrectorado de Investigación de la Universidad de Cantabria. $\tan$ a 1970 con los trabajos preliminares de M.A. García Guinea (1975) y fueron continuadas por nosotros a partir de 1972. Es pues comprensible que, tras más de un cuarto de siglo, hayan cambiado muchas cosas, tanto en lo que respecta a técnicas de registro y datación como al modelo teórico aplicado al estudio de grupos depredadores. Muy especialmente ha entrado en crisis la tendencia «periodizadora» basada exclusivamente en asociaciones de utensilios y en fósiles directores. Tampoco hay que olvidar los problemas terminológicos, ya que muchas de las distinciones horizontales son puramente de nombre y sus diferentes acepciones sólo se entienden en el marco de las distintas tradiciones científicas.

A lo largo de diferentes memorias de excavación e interpretaciones, que han sido sintetizadas hasta la última campaña de 1986 (Moure Romani1lo, 1989,1990), se ha prestado atención al estudio de formas de vida de los ocupantes del área de estancia de la cueva, ubicada en la antigua entrada -hoy taponada por un derrumbe- y a su relación con las evidencias parietales del sector occidental, en especial con su famoso panel polícromo (Balbín Behrmann y Moure Romanillo, 1982; Balbín Behrmann, 1989). El equipo investigador entiende que esos objetivos han sido aceptablemente alcanzados, pero también que las actuaciones se han limitado a la parte superficial de un depósito de 


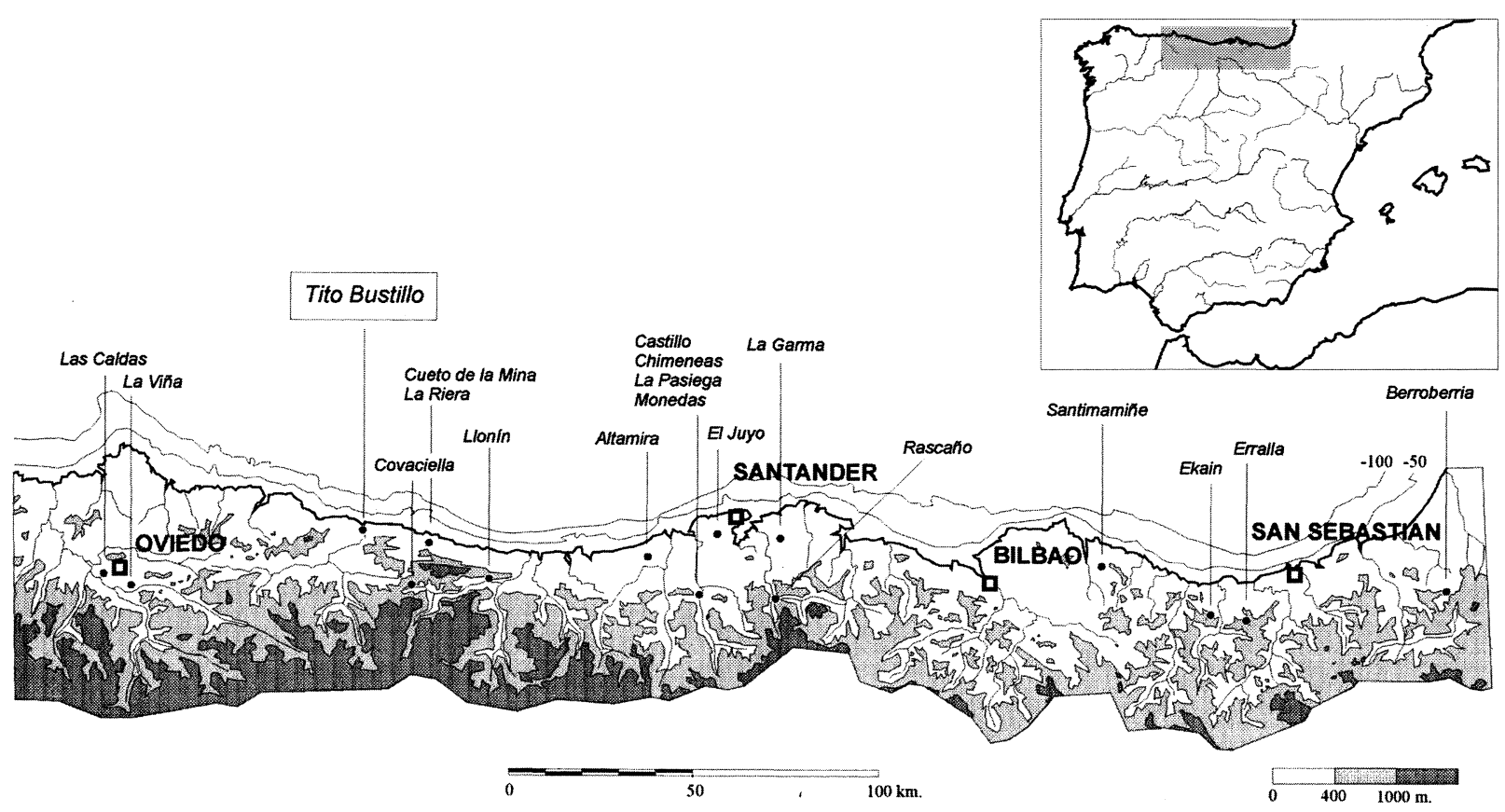

Fig. 1. Localización de la cueva de Tito Bustillo (Ribadesella, Asturias) y otros yacimientos magdalenienses cantábricos mencionados en el texto.

bastante espesor y, en teoría, de alto potencial arqueológico.

Los resultados de la nueva serie de dataciones absolutas por acelerador obtenidos en el Research Laboratory for Archaeology de la Universidad de Oxford permiten replantear la cronología de las últimas ocupaciones magdalenienses de la cueva de Tito Bustillo, algunos de los planteamientos teóricos sobre las industrias tardiglaciales del Cantábrico y, por extensión, el horizonte asimilado al apogeo del hecho artístico.

\section{CARACTERIZACIÓN Y PROBLEMAS CRONOLÓGICOS}

Hasta el momento, la atribución temporal de las zonas de actividad de Tito Bustillo (área de estancia y área de decoración) procedía de la clasificación de la industria, de la evidencia medioambiental y de dataciones absolutas obtenidas por C14 convencional y arqueomagnetismo. En el área de estancia se llevó a cabo un registro extensivo que permitió localizar dos niveles ( 1 y 2), el primero de los cuales ha sido subdividido en ocho capas (1a, 1b, 1ab, 1bc, 1c.1, 1c.2, 1c.3 y 1c.4). El nivel 2 se corresponde con una etapa de inundación presente en posición similar en otras cuevas cantábricas, y por ahora es arqueológicamente indeterminable. A partir del estudio de sus industrias, en el nivel 1 hemos diferenciado dos complejos: el superior (desde 1a hasta 1c.1) y el inferior (desde 1c.2 hasta 1c.4) (Fig. 2).

La industria lítica y ósea responde a los cánones habituales en el Magdaleniense Reciente, si bien, junto a arpones de una fila de dientes, se observa una inusual presencia de utensilios en hueso y obras de arte mueble que la periodización tradicional, heredada de $\mathrm{H}$. Breuil, atribuía a estadios anteriores: varillas semicilíndricas, azagayas de base ahorquillada, azagayas cortas y gruesas con acanaladuras y bisel simple con trazos oblicuos o en espiga (tipo Laugerie-Haute), etc. De ahí que en todos los casos se haya hablado de un horizonte «antiguo» o «arcaico» del Magdaleniense Superior (Moure Romanillo, 1989: 428, 1990: 121-122, 1995a: 243 y 1995b: 29-32). Ya en su tesis doctoral, C. González Sainz por un lado se mostraba, por un lado, justificadamente escéptico ante los resultados de las dataciones $\mathrm{C} 14 \mathrm{y}$, por otro, apuntaba las diferencias entre las capas $1 \mathrm{a} / 1 \mathrm{~b}$ y 1c. Las primeras encajarían sin dificultad en el Magdaleniense Superior, mientras que 1c pertenecería a un Magdaleniense Medio con los atributos característicos

T. P., 54, n. $^{\circ} 2,1997$ 


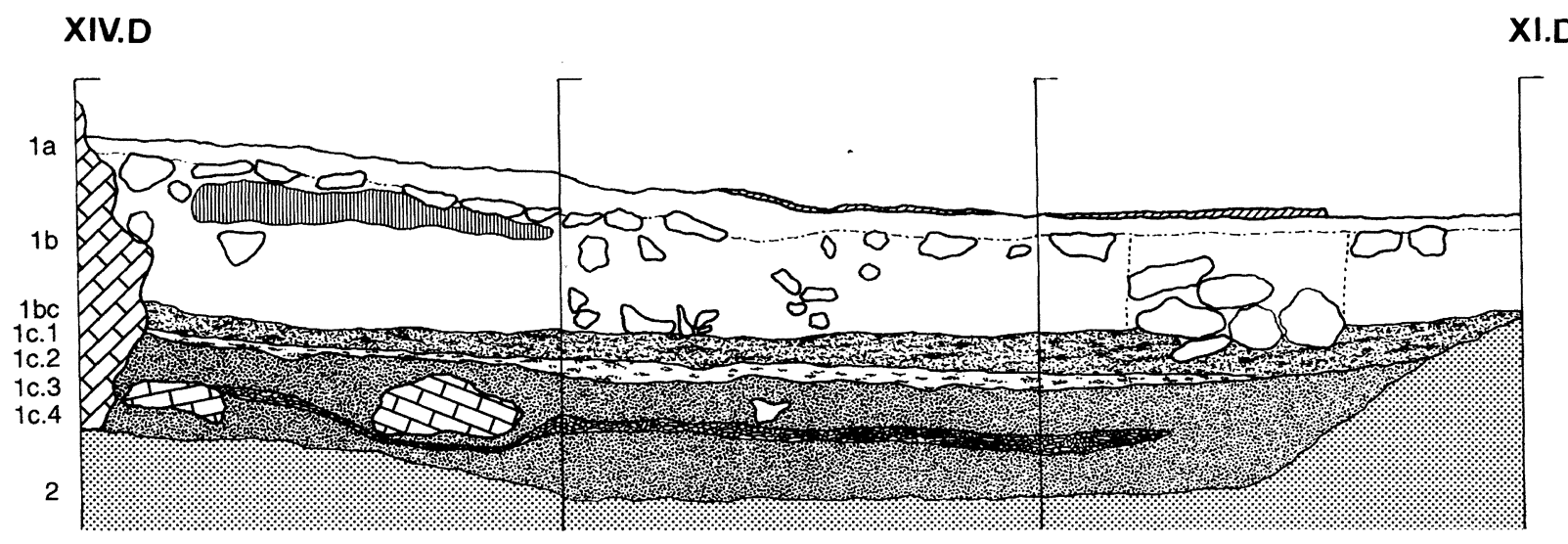

Fig. 2. Estratigrafía de la cueva de Tito Bustillo (Ribadesella, Asturias).

del ámbito pirenaico (González Sainz, 1989: 3546). Sobre un consenso de fondo con esta posición, por nuestra parte debemos señalar que la ampliación de la superficie excavada y, por tanto, de la cantidad de material, tiende a atenuar las diferencias entre el complejo superior y el inferior (Moure Romanillo, 1990: 110-112).

La información medioambiental procedente de los análisis polínicos indica el paso de una fase climática húmeda (el nivel 2) a otra extremadamente fría (nivel 1). La primera se caracteriza por el predominio del paisaje de bosque de pino, especie a la que siguen el aliso y algunos abedules y termófilas (avellano, encina). A lo largo de las diferentes capas del nivel 1, el paisaje estepario con brezos y un progresivo incremento de gramíneas a costa de las ericáceas refleja el desarrollo de un periodo frío. Este enfriamiento se hace especialmente riguroso en la muestra 12 (capa $1 b$ ), en que la reaparición del sauce y el pino viene acompañada de los porcentajes máximos de pólenes de gramíneas (Boyer-Klein, 1976: 205).

A falta de una secuencia estratigráfica más larga y de un estudio sedimentológico que confirme o modifique los resultados del muestreo polínico, la única atribución geoarqueológica disponible es la publicada por A. Boyer-Klein. Para ella el nivel 2 y las diferentes capas del nivel 1 corresponderían a la sucesión Bölling-Dryas II de la secuencia palinológica tardiglaciar, si bien la propia autora del diagrama reconoce que la atribución a esos dos momentos y no a otros de características climáticas similares se basa en la clasificación arqueológica (entiéndase, en la presencia de arpones) (Boyer-Klein, 1980: 106), lo que no es precisamente una evidencia medioambiental. El estudio de la fauna terrestre del nivel 1 indica asimismo que estamos en un periodo de clima frío con ungulados de espacios abiertos, presencia esporádica del reno y micromamíferos como Microtus aeconomus.

\section{LAS DATACIONES ABSOLUTAS}

Uno de los problemas pendientes reside en las dataciones absolutas del nivel 1 , obtenidas hasta ahora por C14 convencional y recopiladas en las últimas síntesis (Moure Romanillo, 1989: 427 y 1990: 121). Algunas de ellas - procesadas en diferentes fechas, laboratorios y a partir de distintos materiales- no son consecuentes con la sucesión estratigráfica, de forma que algunas de las capas superiores han proporcionado fechas más antiguas que otras depositadas con anterioridad. Por otra parte, en su conjunto resultan más antiguas que la de cualquier otro contexto con arpones (y que por ello habrían sido adscritas al Magdaleniense Superior) fechado hasta el presente. «Aceptar» estos resultados nos llevaría a la época del episodio climático Cantábrico V de la secuencia sedimentológica elaborada por M. Hoyos (1995), espacio que en el lenguaje de la Palinología comprendería el «inter» Angles-Prebölling (2) (Tab. 1).

Estas contradicciones nos animaron a remitir para su datación AMS (Accelerator Mass Spectrometry) una serie de 5 muestras procedentes de otras tantas capas del nivel 1 del área de estancia

(2) Debido a un error material en la base de datos utilizada, la datación GrN 12753 (14930 \pm 70 BP.) fue atribuida a la capa 1 b en las memorias referenciadas como Moure Romanillo 1989, 1990 y posteriores. La muestra procedía de un hogar completo (1.240 gr. de carbón vegetal) localizado en superficie de la 1c.2 durante la campaña de 1983. La referencia bibliográfica correcta es la primera publicada (Moure Romanillo, 1985: 113). 


\begin{tabular}{|l|l|l|l|l|l|}
\hline Ref. & Sigla & Material & Nivel & Ref. Laboratorio & Edad BP \\
\hline TB2 & TB72 & Carbón vegetal & $l a$ & CSIC 154 & $14250 \pm 300$ \\
\hline TB3A & TB72 & Caparazones & $l a$ & CSIC 155A & $15180 \pm 300$ \\
\hline TB3B & TB72 & Caparazones & $l a$ & CSIC 155B & $15400 \pm 300$ \\
\hline TB6 & TB74 & Carbón vegetal & $l a$ & CSIC 261 & $14220 \pm 180$ \\
\hline TB4 & TB74 & Hueso & $l c$ & I 8331 & $13870+220$ \\
\hline TB5 & TB74 & Hueso & $I c$ & I 8332 & $13520 \pm 220$ \\
\hline TB8 & TB83 & Carbón vegetal & $l c .2$ & GrN 12753 & $14930 \pm 70$ \\
\hline TB9 & TB86 & Hueso & 2 & Ly 4212 & $14890 \pm 410$ \\
\hline TB10 & TB84 & Hueso & Panel & Ly 3476 & $12890 \pm 530$ \\
\hline TB1 & TBP70 & Carbón vegetal & Panel & CSIC 80 & $14350 \pm 300$ \\
\hline TB7 & TBP72 & Paleomagnetismo & Panel & Kopper, 1973 & 14800 \\
\hline & TBP72 & Paleomagnetismo & Panel & Kopper, 1974 & 11200 \\
\hline
\end{tabular}

Tabla 1.

(tres del complejo superior y dos del inferior) y una más del área de decoración (TBA-1). Los análisis fueron efectuados en la Radiocarbon Accelerator Unit del Research Laboratory for Archaeology de la Universidad de Oxford. En todos los casos fueron utilizadas micromuestras de huesos con marcas de descarnado de localización estratigráfica y topográfica perfectamente documentada. Los resultados obtenidos se recogen en la tabla 2.

Las dataciones obtenidas por acelerador en el área de estancia son, en esta ocasión, consecuentes con la secuencia estratigráfica. Sin embargo, no por ello dejan de plantear algunos interrogantes que sólo podrán ser resueltos mediante futuras investigaciones en éste y en otros yacimientos cantábricos y franceses.

\section{DISCUSIÓN}

La datación AMS de la capa «b» del complejo superior (1b)(OxA 6259, $12850 \pm 90 \mathrm{BP})$ no coincide con las obtenidas en las primeras campañas mediante C14 convencional. Aunque se acerca más a las fechas obtenidas hasta ahora en contex- tos del Magdaleniense con arpones normalizados, seguimos encontrando en ella un amplio porcentaje de utensilios óseos considerados «característicos» del Magdaleniense Medio (varillas semicilíndricas, incluso azagayas de base ahorquillada).

Las fechas AMS de 1bc, 1c. $1,1 c .2$ y 1c. 3 , que van desde el 14550 al 14910 BP, no sólo supondrían la ruptura en una secuencia que hasta ahora se consideraba bastante continua, sino que, nuevamente, nos sitúan ante una antigüedad inusual para un nivel que también incluye un arpón de una fila de dientes. Dado que la ampliación del área excavada ha incorporado nuevas capas y «bolsadas» de extensión limitada, conviene recordar que lo nominado como 1c en las primeras campañas (Moure Romanillo, 1975) se corresponde ahora con las capas 1c. 2 y 1 c. 4 .

Con la única excepción de los arpones (10 en el complejo superior y 1 en el inferior), la industria ósea de cualquiera de los complejos, con abundantes varillas semicilíndricas e incluso azagayas de base ahorquillada, encaja perfectamente en la descripción clásica del Magdaleniense Medio pirenaico. En los últimos años, conjuntos similares han sido descubiertos en Asturias, acompañados

\begin{tabular}{|c|l|l|l|l|l|l|}
\hline Ref. & Sigla & Material & Cuadro & Nivel & OxA- & Edad BP \\
\hline TBA-1 & TBP 71/84 & H marcas & $14 \mathrm{c} / \mathrm{s} 2$ & $\mathrm{a} / \mathrm{d}$ & 6258 & $13520 \pm 110$ \\
\hline TBA-2 & TB-81 & H marcas & XII-C & $1 b$ & 6259 & $12850 \pm 90$ \\
\hline TBA-3 & TB-83 & H marcas & XI-B & $1 b c$ & 6260 & $14550 \pm 110$ \\
\hline TBA-4 & TB-83 & H marcas & XI-B & $1 c .1$ & 6261 & $14440 \pm 100$ \\
\hline TBA-5 & TB-82 & H marcas & XII-C & $1 c .2$ & 6262 & $14680 \pm 110$ \\
\hline TBA-6 & TB-77 & H marcas & XIII-D & $1 c .3$ & 6858 & $14910 \pm 110$ \\
\hline
\end{tabular}

Tabla 2.

T. P., 54, n. ${ }^{\circ} 2,1997$ 
además por los objetos de arte mueble más característicos de este mundo: rodetes, contornos recortados, esculturas, placas grabadas, etc. En alguno de esos casos, como la unidad superior del nivel IX $c$ de la cueva de Las Caldas, clasificada inicialmente como Magdaleniense Medio «evolucionado» se señalan posibles protoarpones que en la base coexisten al menos con dos arpones normalizados.

En el texto antes citado, en que clasificaba la capa 1c de las primeras campañas de Tito Bustillo en el Magdaleniense Medio, C. González Sainz ya apuntaba que la atribución a este episodio o al superior «quizá sea tan sólo cuestión de denominación» ya que «nada impide la aparición en el mismo contexto de protoarpones o de piezas que ya deban considerarse como auténticos arpones» (González Sainz, 1989: 46).

Posiblemente este sea el momento de volver al problema terminológico apuntado al comienzo de este texto. Desde la síntesis de González Echegaray en los años 60, en el Cantábrico el término Magdaleniense III se hizo igual a Magdaleniense Inferior, mientras que en los Pirineos y Aquitania las fases III y IV se asimilaban al Magdaleniense Medio, reservando para el inferior las fases I y II de Breuil.

Esta confusión se evidencia en la práctica diaria de la investigación cantábrica, porque no deja de ser sorprendente que lo que en Asturias se presenta como Magdaleniense Medio se parezca bastante -y presente cronologías absolutas similares- a lo que en Cantabria se está llamado Magdaleniense III o Inferior (p.e. La Viña y Las Caldas por un lado y El Juyo por otro). En el otro extremo de una hipotética discusión sobre la secuencia lineal, habría que reconocer la semejanza de la industria ósea (para la lítica no disponemos aún de información suficiente) entre el llamado Magdaleniense Medio de varios yacimientos en estudio del valle del Nalón y todas las capas del nivel 1 de Tito Bustillo.

$\mathrm{Si}$ en este caso puede hablarse de responsables, no creo que ninguno de los investigadores de la región podamos considerarnos libres de culpa. En lo que respecta al proyecto Tito Bustillo, debo reconocer que si en la zona excavada en nuestras campañas no hubiera aparecido ningún arpón, no hubiéramos pretendido ni conseguido mucho más que ampliar y matizar la clasificación efectuada por M.A. García Guinea (1975) como Magdaleniense Medio ( «Magdaleniense III» en aquellos primeros trabajos) y extender su cronología al arte rupestre como entonces hicieron Almagro Basch, García Guinea y Berenguer Alonso (1972). En nuestro caso las asignaturas pendientes siguen siendo la antigüedad del arpón de 1c y-limitándonos a los resultados por AMS- la discontinuidad entre el complejo inferior y el superior, que entraría en contradicción con la semejanza de su industria ósea, que integra objetos no muy frecuentes en el Cantábrico. En lo que concierne al arpón de 1c podemos decir que, aunque presenta una morfología bastante atípica (Moure Romanillo, 1990: 113, Fig. 6-4) -por lo que en algún caso fue considerado una variedad tipológica (Cano Herrera, 1977)-, cuenta con una hilera de dientes exentos, por lo que sin lugar a dudas es un arpón normalizado que no puede ser clasificado como protoarpón.

Las dataciones de contextos con arpones anteriores al 13000 son escasas y, en ocasiones, problemáticas (Teufelsbrücke, Kniegrotte, Verlaine, Kents Cavern, Pierre Chatel y Rond Barry (Julien, 1982: 201). La capa IX de Le Flageolet se ha convertido en referencia clásica de industrias líticas clasificadas en el Magdaleniense Superior con dataciones por encima del 14000 (Rigaud, 1976: 1269 y 1979: 467) que se corresponden con el «episodio Saiga» (Delpech et alii, 1970: 223).

\section{REFLEXIÓN FINAL}

Los resultados en las excavaciones de Tito Bustillo nos sitúan ante un grupo cultural de cazadores especializados en el acoso de manadas de ciervos que comenzaban a prestar atención a otros recursos costeros (fundamentalmente moluscos de roquedo) y fluviales. Los conjuntos de artefactos presentes en las distintas capas con que los aportes naturales $y / o$ humanos han configurado la estratigrafía del nivel 1 no presentan diferencias que puedan ser consideradas culturalmente significativas. Cualquiera que haya dirigido una excavación y estudiado posteriormente los materiales recogidos puede dar testimonio de que la composición de esos conjuntos difiere sensiblemente -sea por variabilidad funcional, sea por azar- entre cuadrículas y sectores limítrofes. Difícilmente puede por ello sostenerse una periodización lineal basada en objetos singulares bajo la acepción tradicional de «fósil director» sin el respaldo de alguna referencia cronológica absoluta o relativa.

Como ejemplo, baste recordar que los arpones constituyen un porcentaje irrelevante del equipo 
material de cualquier yacimiento. En el caso del complejo superior de la cueva de Tito Bustillo, que no puede ser considerado precisamente pobre en industria en hueso o asta, éstos sólo suponen en torno al 5 por ciento de los artefactos óseos y menos de un 0,5 por ciento del total de útiles «tipológicos». En el complejo inferior hasta el momento sólo ha sido localizado un arpón, de forma que cuantitativamente ni siquiera puede ser tenido en cuenta. Por el contrario, si nos fijamos en instrumentos más cotidianos (azagayas, varillas, agujas, punzones) y en los índices tipológicos de la industria lítica, parece que a lo largo de todas las capas del nivel 1 hay bastante continuidad en la fabricación y uso de su equipo material, continuidad que sin duda podemos hacer extensiva a todo el tecnocomplejo.

Las dataciones absolutas insisten de manera francamente testaruda en llevar el nivel 1 inferior de Tito Bustillo más allá del 14000 BP. Su paralelo más inmediato es el ya mencionado nivel IXc de Las Caldas, que también se superpone a la inundación representada en el nivel X, arqueológicamente estéril, que Hoyos sitúa en su fase Cantábrico IV (c. Prebölling en la secuencia polínica) (Hoyos Gómez, 1995: 47).

Ciertamente, las nuevas dataciones aportan una solución de continuidad entre $1 \mathrm{~b}$ y las capas $1 \mathrm{bc}$ e inferiores que, por ahora, no ha podido ser contrastada sedimentológicamente. A pesar de la ya señalada persistencia de útiles y objetos de arte mueble del Magdaleniense Medio pirenaico -en este sentido está plenamente justificada la atribución cronológica de González Sainz (1994: 61)creemos que ahora estamos en condiciones de defender una antigüedad por encima del $14000 \mathrm{BP}$ para las capas $1 \mathrm{bc}, 1 \mathrm{c} .1,1 \mathrm{c} .2,1 \mathrm{c} .3$ y $1 \mathrm{c} .4$. Ya que no podemos avanzar más de lo que los datos disponibles nos permiten, incorporando la nueva datación de 1b (OxA 6259: 12850 \pm 90) a lo largo de todo el nivel 1 nos encontraríamos dentro del espacio tardiglaciar ocupado en el Cantábrico por cazadores especializados en ciervos cuyas industrias encajan -sin que sus autores fueran conscientes de tal situación-en el tecnocomplejo conocido como Magdaleniense Reciente.

La datación OxA $6258(13520 \pm 110 \mathrm{BP})$ procedente del área de decoración queda dentro del intervalo delimitado por las fechas anteriormente obtenidas en el mismo lugar tanto en las excavaciones de García Guinea (1975) como durante la recogida y revisión de los materiales (Moure Ro- manillo y González Morales, 1988,1989). El problema de fondo procede aquí de la propia fiabilidad del registro, que impide garantizar si existe un único depósito sincrónico o que, por el contrario, los restos aluden a distintas fases de la ejecución del gran panel. No obstante estas evidencias descansan sobre un lecho de inundación que en el futuro habrá que comprobar si, como parece, se corresponde con nuestro nivel 2, lo que apuntalaría su correlación con el área de estancia.

En el marco cronológico establecido para las dos zonas de actividad humana de la cueva (áreas de estancia y de decoración) se sitúan la mayor parte de los grandes santuarios y superposiciones de arte rupestre. Para éstos, y concretamente para los grandes paneles con polícromos, hemos defendido la continuidad desde el horizonte de pinturas negras y grabados de ciervas «tipo Altamira-El Castillo» hasta el final de los polícromos (Moure Romanillo y Bernaldo de Quirós, 1995; Moure Romanillo, 1995a,b). Dicho de otra manera, que las superposiciones mencionadas tienen un significado en símismas, sin que haya sido necesario el transcurso de milenios entre una fase de decoración y otra. En Altamira la datación directa de pigmentos mediante análisis por AMS ha proporcionado fechas que también remontan ampliamente el $14000 \mathrm{BP}$ en los polícromos, en uno de los famosos omoplatos decorados y en un trazo negro infrapuesto a su paralelo parietal más evidente (Moure Romanillo et alii, 1995).

En síntesis, los resultados de esta nueva serie de dataciones vienen a reforzar la cronología «larga» del nivel 1 de Tito Bustillo, lo que una vez más permite cuestionar el esquema unilineal de evolución de las industrias magdalenienses y el modelo teórico de las Ciencias Naturales en que se inspira. $\mathrm{La}$ correspondencia entre la frecuentación del área de estancia de esta cueva asturiana y las fases de decoración de su panel principal -o, al menos, de la «serie reciente»- está acreditada por numerosos argumentos expuestos en trabajos anteriores. Las fechas del complejo inferior coinciden casi literalmente con las obtenidas en Altamira para la parte de las superposiciones antes citada, aunque estas últimas puedan haberse visto rejuvenecidas por la circulación de agua por el techo y la proximidad de este a la superficie exterior. Nuevamente en Tito Bustillo, por encima de los polícromos, pero conservando profundas analogías con ellos, se encuentran figuras tan elocuentes como el equino número 40 (Balbín Behrmann y Moure Romanillo, 
1982), que es perfectamente paralelizable con una de las plaquetas de-coradas de la capa 1b (Moure Romanillo, 1990: Fig. 9,2).

Entre 15000 y 13000/12500 BP, época de apogeo del arte mueble y parietal, hay una frecuentación continuada de ciertos yacimientos paralela a la decoración de sus paneles con superposiciones que reproducen secuencias similares. Las diferencias entre los conjuntos de artefactos que aparecen asociados a un grupo cultural de cazadores especializados correspondiente a ese tramo cronológico se deben a la variabilidad funcional, al azar o a ambas cosas. Y, desde luego, la presencia o ausencia de algunos objetos singulares, ya sean arpones o contornos recortados, es irrelevante frente a las evidencias de su caracterización cultural.

\section{BIBLIOGRAFÍA}

Almagro Basch, M.; García Guinea, M.A. y Berenguer Alonso, M. (1972): «La época de las pinturas y esculturas cuaternarias polícromas en relación con los yacimientos: revalorización del Magdaleniense III». En Santander Symposium. UISPP-Patronato de las Cuevas Prehistóricas de la Provincia de Santander. Madrid: 467-474.

Balbín Behrmann, R. de (1989): «L'art de la grotte de Tito Bustillo (Ribadesella, Espagne). Une vision de synthèse». L'Anthropologie, 93-2: 435-462.

Balbín Behrmann, R. de y Moure Romanillo, A. (1982): «El panel principal de la cueva de Tito Bustillo». Ars PraehistoRICA, 1: 47-94.

Boyer-KLEIN, A. (1976): «Análisis polínico de la cueva de Tito Bustillo (Asturias)». En A. Moure Romanillo y H. Cano Herrera: Excavaciones en la cueva de «Tito Bustillo» (Asturias). Instituto de Estudios Asturianos. Oviedo: 201-207.

- (1980): «Nouveaux résultats palynologiques de sites solutréens et magdaléniens cantabriques». Bulletin de la Societé Préhistorique Française, 77-4: 103-107.

Cano Herrera, M. (1977): «Hueso trabajado en el Magdaleniense Superior de la región cantábrica: nuevos tipos de arpón procedentes dela cueva de Tito Bustillo». Actas del XIV Congreso Nacional de Arqueología (Vitoria, 1975). Zaragoza: 201-214.

Delpech, F.; Laville, H. et Rigaud, J.PH. (1976): «Magdalénien 'Moyen' ou Magdalénien 'Superieur'». En H. de Lumley (ed.): IX Congrés de l'UISPP (Niza): 223.

García Guinea, M.A. (1975): Primeros sondeos estratigráficos en la cueva de Tito Bustillo (Ribadesella, Asturias). Excavaciones de 1970. Publicaciones del Patronato de las Cuevas Prehistóricas de la Provincia de Santander, XII. Santander.
GonZÁlez SAInZ, C. (1989): El Magdaleniense SuperiorFinal en la Costa Cantábrica. Editorial Tantín. Santander.

- (1994): «Sobre la cronología del Magdaleniense y Aziliense en la región cantábrica». Munibe, 56: 53-68.

- (1995): «13.000-11.000 BP. El final de la época Magdaleniense en la región Cantábrica». En A. Moure Romanillo y C. González Sainz (eds.): El final del Paleolítico Cantábrico. Transformaciones ambientales y culturales durante el Tardiglaciar y comienzos del Holoceno en la Región Cantábrica. Universidad de Cantabria. Santander: 159-198.

Hoyos GonZÁLEZ, M. (1995): «Cronoestratigrafía del Tardiglaciar en la región cantábrica». En A. Moure Romanillo y C. González Sainz (eds.): El final del Paleolítico Cantábrico. Transformaciones ambientales y culturales durante el Tardiglaciar y comienzos del Holoceno en la Región Cantábrica. Universidad de Cantabria. Santander: 15-75.

JuLIEN, M. (1982): Les harpons magdaléniens. Centre National de la Recherche Scientifique. París.

Moure Romanillo, A. (1975): Excavaciones en la cueva de "Tito Bustillo» (Asturias)(Campañas de 1972 y 1974). Instituto de Estudios Asturianos, Oviedo.

- (1985): «Nouveautés dans l'art mobilier figuratif du Paléolithique cantabrique». Préhistoire Ariégeoise, XXXX: 99-130.

- (1989): «La caverne de Tito Bustillo (Asturias, Espagne). Le gisement paléolithique». L'Anthropologie, 93-2: 407-434.

- (1990): «La cueva de Tito Bustillo (Ribadesella, Asturias). El yacimiento paleolítico». Excavaciones Arqueológicas en Asturias 1983-86. Junta del Principado de Asturias. Oviedo: 107-127.

- (1995a): «Después de Altamira: transformaciones en el hecho artístico al final del Pleistoceno». En A. Moure Romanillo y C. González Sainz (eds.): El final del Paleolítico Cantábrico. Transformaciones ambientales y culturales durante el Tardiglaciar y comienzos del Holoceno en la Región Cantábrica. Universidad de Cantabria. Santander: 225-257.

- (1995b): «Características culturales y económicas del final del Paleolítico Superior en la Región Cantábrica». En V. Villaverde Bonilla (ed.): Los últimos cazadores. Transformaciones culturales y económicas $d u$ rante el Tardiglaciar y el inicio del Holoceno en el ámbito mediterráneo. Instituto de Cultura Juan GilAlbert. Alicante: 23-43.

Moure Romanillo, A. y Bernaldo de Quirós, F. (1995): «Altamira et Tito Bustillo, réfléxions sur la chronologie de l'art polychrome à la fin du Paléolithique Supérieur». L'Anthropologie, 99, 2-3: 286-295.

Moure Romanillo, A. y González Morales, M.R. (1988): «El contexto del arte parietal. La tecnología de los artistas en la cueva de Tito Bustillo (Asturias)». Trabajos de Prehistoria, 45: 19-49. 
Moure Romanillo, A. y González Morales, M.R. (1989): «Le contexte de l'art pariétal: la technologie des artistes». En L'Art Pariétal Paléolithique (Périgueux-Le Thot, 1984). Ministère de la Culture. Clamecy: 407-434.

Moure Romanillo, A.; González Sainz, C.; Bernaldo de Quirós, F. y CABRERA VALDÉs, V. (1996): «Dataciones absolutas de pigmentos: Altamira, El Castillo, Chimeneas y Las Monedas». En A. Moure Romanillo (ed.): «El hombre fósil» 80 años después. Universi- dad de Cantabria, Fundación Marcelino Botín, Institute for Prehistoric Investigations. Santander: 295324.

Rigaud, J.Ph. (1976): «Les civilisations du Paléolithique Supérieur en Périgord». En H. de Lumley (dir.): $L a$ Préhistoire Française, I-2. CNRS. París: 1257-1270.

- (1979), «A propós des industries magdaléniennes de Flageolet». En D. de Sonneville-Bordes (ed.): La Fin des Temps Glaciaires en Europe (Talence, 1977). Colloque International du CNRS 271. París: 467-469.
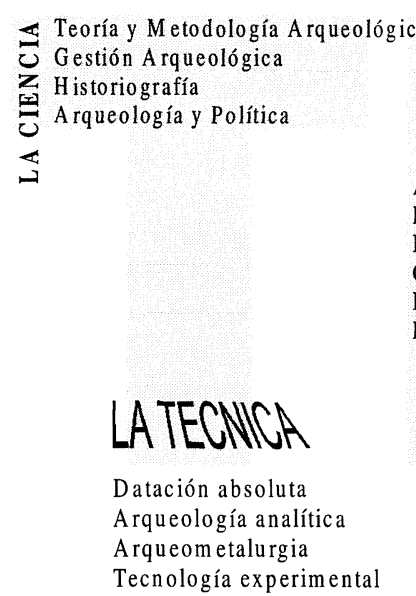

$$
\mathrm{L}^{\mathrm{A}} \mathrm{CULT}_{\mathrm{R}_{\mathrm{A}}}
$$

Arqueología Prehistórica y Protohistórica Paleolítico

Neolítico

Calcolítico

Edad del Bronce

Edad del Hierro

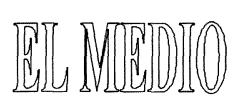

A rqueobotánica A rqueozoología A rqueología del Paisaje

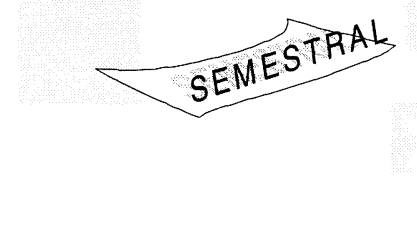

Trabajos de Prehistoria es la revista de consulta imprescindible para todos aquellos interesados en conocer el estado de la cuestión sobre el rico patrimonio arqueológico de la Prehistoria y Protohistoria de la Península Ibérica. Sus páginas reflejan tendencias punteras de su especialidad, por lo que figura en los más significativos repertorios bibliográficos nacionales e internacionales.

\section{TRABAJOS DE PREHISTORIA}

\section{BOLETIN DE PEDIDO

$$
\begin{aligned}
& \text { neje } \\
& \text { CSIC } \\
& \text { CSIC }
\end{aligned}
$$ \\ Servicio de Publicaciones \\ Teléfono: $91 / 5612833$ Fax: $91 / 5629634$ Vitruvio, 8 - 28006 Madrid (España)

Nombre
Dirección
Ciudad

Adjunto cheque bancario por valor de a nombre de Servicio de Publicaciones CSIC

Contra reembolso

Envíenme, por favor, factura pro-forma Tarjeta $V$ isa $\mathrm{N}^{\circ}$

Fecha de caducidad

\section{Suscripción anual}

Fecha.

Firm a
(Revista Semestral)

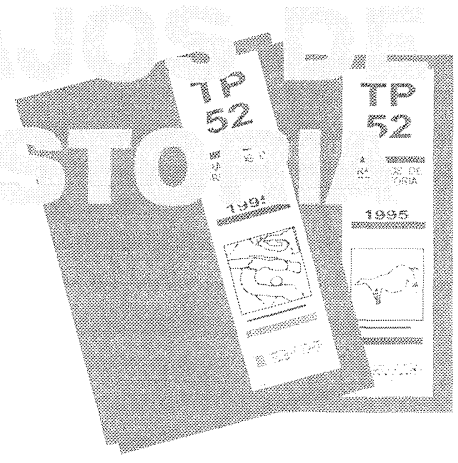

